Copyright by the Cambridge University Press. Nair, A. K.; Cordill, M. J.; Farkas, D.; Gerberich, W. W.,

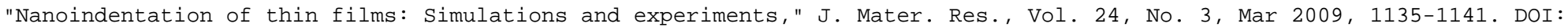
$10.1557 / \mathrm{jmr} .2009 .0136$

\title{
Nanoindentation of thin films: Simulations and experiments
}

\author{
Arun K. Nair ${ }^{\text {a) }}$ \\ Department of Engineering Science and Mechanics, Virginia Tech, Blacksburg, Virginia 24061 \\ M.J. Cordill ${ }^{\text {b) }}$ \\ Department of Chemical Engineering/Materials Science and Engineering, University of Minnesota, \\ Minneapolis, Minnesota 55455 \\ Diana Farkas ${ }^{\text {c) }}$ \\ Department of Materials Science and Engineering, Virginia Tech, Blacksburg, Virginia 24061 \\ W.W. Gerberich \\ Department of Chemical Engineering/Materials Science and Engineering, University of Minnesota, \\ Minneapolis, Minnesota 55455
}

(Received 23 July 2008; accepted 20 November 2008)

\begin{abstract}
Atomistic simulations of nanoindentation of a 20-nm-thick Ni thin film oriented in the [111] direction were carried out to study the effects of indenter velocity and radii, interatomic potentials, and the boundary conditions used to represent the substrate. The simulation results were compared directly with experimental results of Ni thin film of the same thickness and orientation. It was found that the high indenter velocity does not affect the hardness value significantly. Different radii used for indentation also have negligible effects on the hardness value. Two different interatomic potentials were tested, giving significantly different hardness values but both within $20 \%$ of the experimental result. Different boundary conditions used to represent the substrate have a significant effect for relatively deep indentation simulations.
\end{abstract}

\section{INTRODUCTION}

One of the most important applications of nanoindentation is the determination of the mechanical properties of thin films. In nanoindentation tests, the properties of the thin film may be measured without removing the film from the substrate as is done in other types of testing. The spatial distribution of properties, in both lateral and depth dimensions, may be measured. Apart from testing films in situ, nanoindentation techniques can also be used for films made as freestanding microbeams or membranes. ${ }^{1}$ Advances in experimental equipment allow the testing to be performed with the indenter radii in the tens of nanometer scale. Experimental studies $^{2}$ have shown that nanoindentation is a reliable and widely used technique for probing mechanical properties of materials. At the same time, computing capabilities now allow for atomistic simulations in parallel architectures that can approach these length scales. These advances make it possible for the first time to actually compare simulation and experimental results in a direct

\footnotetext{
a) Present address: Cornell University, Ithaca, NY 14853.

b) Present address: Erich Schmid Institute for Materials Science, Austrian Academy of Sciences, Leoben, Austria 8700.

${ }^{c)}$ Address all correspondence to this author.

e-mail: diana@vt.edu

DOI: 10.1557/JMR.2009.0136
}

manner. A simulated load or pressure versus depth curve allows comparisons to be made with actual experimental data. These direct comparisons can be used to shed light on basic unresolved questions such as the effects of the unrealistically fast indenter speeds typically used in simulations, and the uncertainties related to the reliability of the interatomic potentials.

Simulations have been used extensively in the past to study the nanoindentation problem and have provided great insight into the actual process of emission of the first dislocations responsible for incipient plasticity as the films are indented. ${ }^{3-10}$ Christopher et al. ${ }^{3}$ have shown that atomistic modeling was able to reproduce the experimental features like piling up of material along the indenter sides. Studies conducted by $\mathrm{Li}$ et $\mathrm{al}^{4}{ }^{4} \mathrm{de}-$ scribed the incipient plasticity due to indentation using atomistic simulation and finite element modeling for Al. Atomistic/continuum modeling of nanoindentation was also carried out by Shiari et al. ${ }^{8}$ and Iglesias and Leiva. ${ }^{9}$ Ma et al. ${ }^{6}$ have also modeled $\mathrm{Al}$ and described in detail the homogenous nucleation of dislocations. Szlufarska ${ }^{11}$ and Schuh ${ }^{12}$ have reviewed recent advances in nanoindentation studies by atomistic modeling. The effects of thickness and geometry of the indenter in nanoindentation of Ni thin films were studied by Parkala et al., ${ }^{13}$ showing that there is an indentation size effect irrespective of indenter tip geometries. Nix and $\mathrm{Gao}^{14}$ have 
modeled indentation size effects at the micro scale using the concept of geometrically necessary dislocations and found that hardness increases as depth decreases. However, the model does not address the hardness-depth relation for indentation at the nanoscale. Huang et al. ${ }^{15}$ developed an analytical model applicable to indentation at the nanometer scale based on maximum allowable geometrically necessary dislocations. Zhang et al. ${ }^{16}$ modeled tungsten films deposited on an aluminum substrate using the conventional mechanism of strain gradient plasticity theory (CMSG), showing that hardness at the microscopic scale decreases monotonically as the indentation depth increases and does not reach a constant macroscopic hardness. Cordill et al. ${ }^{17}$ have used experiments and simulations of indentation of a nickel thin film to describe the mechanism of staircase yielding. Chen et al. ${ }^{18}$ studied the size dependence in $\mathrm{Al}$ films on glass and showed that the hardness increases when the tip approaches the film/substrate interface. Recently, Li et al. ${ }^{19}$ used the finite element method (FEM) to investigate the load-displacement behavior of the hardness of the hard coating/interlayer/soft substrate system during nanoindentation. Using molecular dynamics simulations, Nair et al. ${ }^{20}$ studied the thickness effects and deformation mechanisms of single crystal $\mathrm{Ni}$ thin films. Khatibi et al. ${ }^{21}$ studied nanoindentation behavior of single crystal silicon using a hybrid model combining molecular dynamics and finite elements. Meza et al. ${ }^{22}$ showed that the penetration depth and tip radius have a dependence on the correction factor (beta) and can be expressed as two multiplicative contributions, a contribution related to the mechanical properties of the material and a contribution related to the indenter geometry. Basu et al. ${ }^{23-25}$ studied the effects of using a spherical indenter in nanoindentation, which included the deformation mechanism of $\mathrm{ZnO}$ single crystals and the determination of the effective zero point of contact.

Large scale massively parallel simulation techniques now allow simulations for film thicknesses and indenter radii that are the same as in experiments. The purpose of the present work is to address the nanoindentation of a 20-nm Ni film using both simulations and experiments. In this work, a standard molecular dynamics (MD) simulation technique is used for direct comparisons. Although the length scale is the same in the simulations and experiments, the simulations are still performed at unrealistically high indenter velocities, as compared with experiments. In addition, questions remain about the empirical nature of interatomic potentials used, and how to represent the substrate properties in the simulations. In the comparison of simulation and experiments presented here we address the following questions.

(i) The effects of unrealistically fast indenter speeds required in MD simulations. (ii) The extent of substrate effects and the different possible boundary conditions that can be used to represent the substrate properties.

(iii) The effects of the indenter radius used, which is typically smaller in the simulations than in the experiments.

(iv) The effects of using different interatomic potentials that are constructed to represent the same material, face-centered-cubic (fcc) Ni in our case.

In the following sections, we describe our simulation and experimental technique and address each one of the previous questions.

\section{SIMULATION SETUP}

A sample of single crystal film was generated for a thickness of $20 \mathrm{~nm}$ oriented in the [111] direction. The dimensions of the sample are $52 \mathrm{~nm}$ along the $[1 \overline{1} 0]$ direction and $52 \mathrm{~nm}$ along the [112] direction (Fig. 1). The sample size is significantly larger than the indentation contact area at maximum depth, and the spurious effects originating from the finite size of the sample in these directions can be avoided. These effects could arise because of the periodic boundary conditions. In particular, the presence of dislocations in the periodic block may cause back stresses that affect the further emission of dislocations in the main block. This in turn would affect the overall observed hardness. These spurious effects can be detected by checking that a further increase in sample size in these periodic directions does not alter the results. For the present samples, we have found that no spurious results occur if there is at least a distance of $15 \mathrm{~nm}$ between the contact region and the boundary of the sample. Before the indentation process, the sample was relaxed for 100 ps at $300 \mathrm{~K}$ using molecular dynamics as implemented in the Largescale Atomic/Molecular Massively Parallel Simulator (LAMMPS) code. $^{26}$ This relaxation was performed with free-surface boundary conditions for the top of the film and three fixed layers of atoms to represent the substrate. The boundary conditions in the directions contained in the film are periodic, using a Nose-Hoover thermostat $(300 \mathrm{~K})$ and barostat (zero pressure).

Two different boundary conditions were used in the directions parallel to the film surface, representing different substrate behavior. First, the boundary condition considers the substrate is infinitely hard and is not allowed to expand at all (BC-1). In the second boundary condition the substrate is represented by atoms that are not allowed to move perpendicular to the film surface but are allowed in-plane expansion (BC-2).

To analyze the influence of rate (velocity of indenter) in nanoindentation, indentation was carried out for two different indenter velocities. In the first case the indenter velocity was chosen as $10 \mathrm{~m} / \mathrm{s}$, and in the second case it 


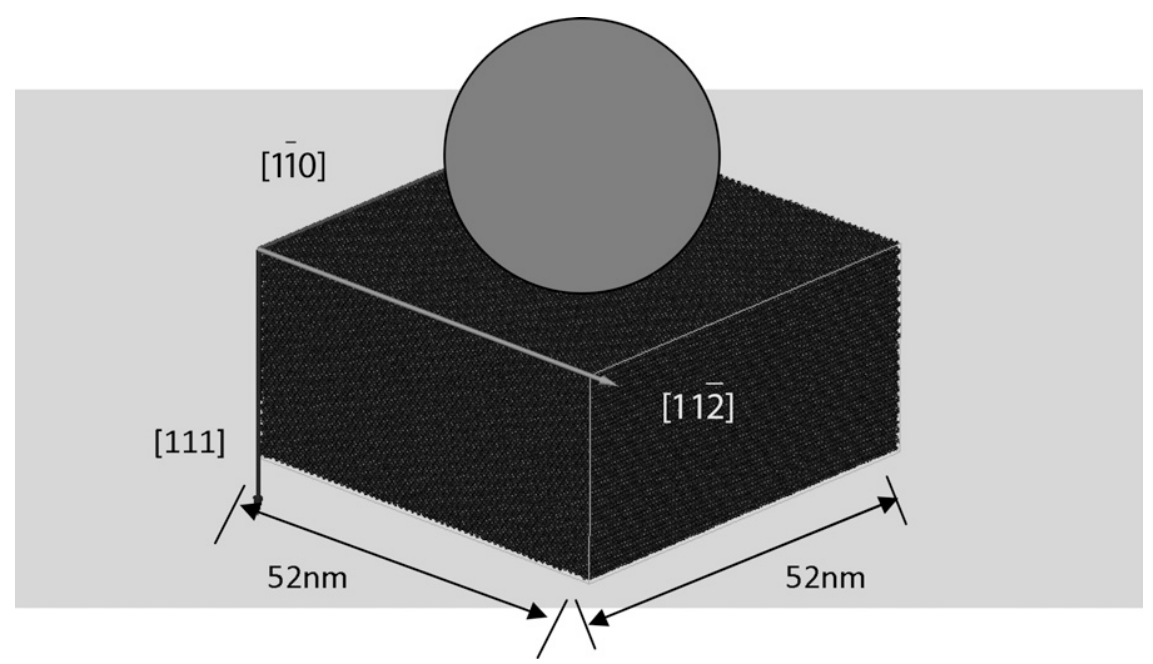

FIG. 1. Orientation and size of the single crystal films used in the indentation simulations.

was four times slower at $2.5 \mathrm{~m} / \mathrm{s}$. Note that both of these velocities are much faster than the experimental ones, as constrained by available computational times.

To study the effect of different interatomic potentials we chose two different embedded atom empirical potentials, one developed by Mishin et al. ${ }^{27}$ and the other by Voter et al. ${ }^{28}$ The Voter potential was used for studying the effects of indenter velocity, indenter tip radius, and type of boundary conditions.

\section{EXPERIMENTAL SETUP}

In the experimental setup 20-nm $\mathrm{Ni}$ film was grown on an $\mathrm{Al}_{2} \mathrm{O}_{3}$ substrate with orientation in the [111] direction. The Ni film was grown to a thickness of 20 $\mathrm{nm}$ and was deposited by molecular-beam epitaxy $(\mathrm{MBE})$ in a system described elsewhere. ${ }^{29}$ This deposition took place at $0.1 \AA / \mathrm{s}$ with a base pressure below $10^{-10}$ Torr, after chemical cleaning of the substrates followed by vacuum annealing. Wide angle $\mathrm{x}$-ray diffraction confirmed the growth orientation and coherence in the growth direction, whereas in-plane diffraction and in situ reflection high-energy electron diffraction (RHEED) confirmed in-plane epitaxy. The quasi-static [direct current (dc) loading] displacement rate was about $1 \mathrm{~nm} / \mathrm{s}(20 \mu \mathrm{N} / \mathrm{s}$ loading rate) with a 50-nm-radius Berkovich tip.

\section{SIMULATION PROCEDURE}

\section{A. Interatomic potentials}

The embedded atom method is currently a common technique used in molecular-dynamics computer simulation of metallic systems. ${ }^{30}$ The method provides a good description of the interatomic forces in the system, particularly for fcc metals. It calculates the interatomic potentials in metals and models forces between atoms as follows:

$$
\begin{aligned}
& E=\frac{1}{2} \sum_{i, j} V\left(r_{i j}\right)+\sum_{i} F\left(\rho_{i}\right) \\
& \rho_{i}=\sum_{j \neq i} \phi\left(r_{i j}\right),
\end{aligned}
$$

where $E$ is the total energy of the system, $V\left(r_{i j}\right)$ represents the pair interaction energy between an atom $i$ and its neighboring atom $j, \varphi\left(r_{i j}\right)$ is the electronic density function, and $F\left(\rho_{i}\right)$ represents an embedding function accounting for the effects of the free electrons in the metal. ${ }^{31}$ It is particularly important to use a potential that can reproduce off-equilibrium situations, because the atomic configurations that are encountered during indentation are under large compressive stresses and therefore far from perfect lattice equilibrium.

The molecular dynamics algorithm is used here as implemented in the LAMMPS code by Plimpton. ${ }^{26}$ The boundary conditions used are periodic in the directions that are perpendicular to the indentation force. In the periodic directions, a Nose-Hoover barostat is used to maintain the pressure in these directions at zero. The temperature is maintained at $300 \mathrm{~K}$ using a Nose-Hoover thermostat. For the indenter, we use a totally rigid spherical indenter with a force on each atom given by ${ }^{26}$ :

$$
F(r)=-k(r-R)^{2},
$$

where $k$ is the specified force constant, $r$ is the distance from the atom to the center of the indenter, and $R$ is the radius of the indenter. The force obtained using Eq. (2) showed excellent agreement with the force computed from pressure in the [111] direction in the elastic regime. 


\section{B. Indentation simulation and visualization of the results}

The indention process was started by lowering a rigid spherical indenter of radius $30 \mathrm{~nm}$ down the [111] direction into the thin film at the rate of $10 \mathrm{~m} / \mathrm{s}$. This rate is much faster than in experiments, and is a result of the limitations of the molecular dynamics technique, even using massively parallel simulations, as we do here. The simulation was performed at $300 \mathrm{~K}$ with the bottom three atomic layers fixed in the [111] direction. The force from the indenter was calculated using Eq. (2), and the atomic positions corresponding to each time step monitored at $10 \mathrm{ps}$ intervals. Finally, the contact area between the indenter and the film needed to obtain contact pressures was calculated as

$$
\text { Area }=\pi\left(2 R \delta-\delta^{2}\right),
$$

where $R$ is the radius of the indenter and $\delta$ is the indentation depth. The areas computed using Eq. (3) have been compared with the areas computed by the direct observation of the indented site. Hence, it is concluded that Eq. (3) gives an accurate area as long as the indentation depth remains small compared to the indenter radius.

To selectively visualize interior defects, we have used the visualization technique based on the centrosymmetry parameter that was proven effective for the studies of dislocation emission in fcc crystals. ${ }^{32}$ Centrosymmetry parameter values between 3 and 6 are typical of dislocation cores and stacking faults. Centrosymmetry parameter values larger than that are typical of atoms at the surface or the interface with the substrate. The atoms with centrosymmetry parameter lower than 3 indicate atoms in the near perfect lattice positions and are not displayed for visualization purposes. Figure 2 shows a typical sequence of emission of dislocations as the indentation proceeds.

\section{RESULTS AND DISCUSSION}

\section{A. Indenter velocity effects}

Molecular dynamics techniques necessarily have to be performed at extremely fast rates. In the present work, we have successfully reached in our simulations the length scales of the experiments but the time scales have not yet been bridged. Therefore, it is important to study the effects of indenter speed on the material hardness obtained. For this purpose we used two different indentation velocities that differ by a factor of four, even if both velocities are unrealistically fast when compared with experiments. Figure 3 shows that these different indenter velocities in the simulation have no significant effect on the thin film in the elastic region and the hardness values. Overall response of the material in this region is not significantly affected by the indentation rates. In our own experiments we have not varied the indenter velocity, but our simulation results are consistent with experimental studies done by Parkala et al. ${ }^{13}$ This can be interpreted in terms of the basic deformation mechanism. In our simulations dislocations emitted under the indenter are mainly responsible for deformation. Figure 2 shows the dislocation formation and propagation during indentation. This deformation mechanism implies a large activation volume and correspondingly low rate sensitivity.

\section{B. Effect of different boundary conditions representing the substrate}

Two boundary conditions studied here using simulations are BC-1 (thin film on a completely fixed substrate, with no in-plane expansion) and $\mathrm{BC}-2$ (thin film on a substrate that allows an in-plane expansion, fixed only in the direction of indentation). BC-1 better represents a perfectly hard substrate, while BC-2 is expected to better represent substrates that can deform to some extent. Figure 4 shows that the use of $\mathrm{BC}-1$ or $\mathrm{BC}-2$ has no effect in the elastic region. However, in the plastic region the

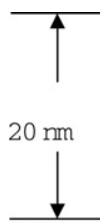

(a)

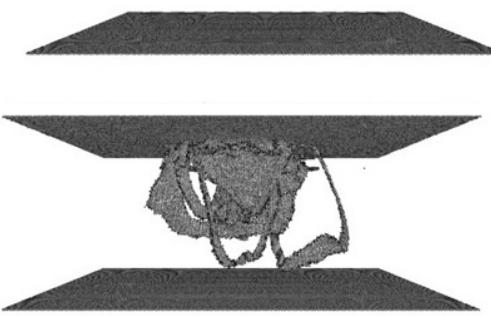

(c)
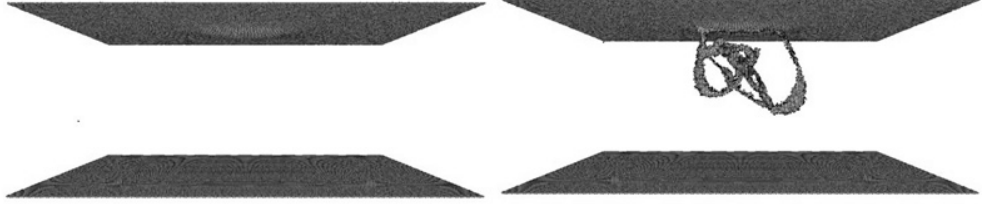

(b)

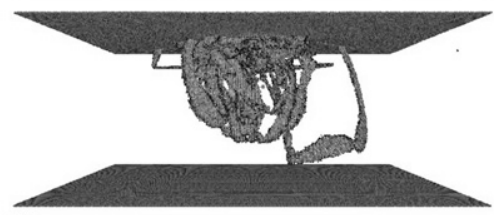

(d)

FIG. 2. Dislocation emission due to indentation of a 20-nm Ni thin film at various depths: (a) $1.4 \mathrm{~nm}$, (b) $1.5 \mathrm{~nm}$, (c) $1.6 \mathrm{~nm}$, and (d) $1.7 \mathrm{~nm}$. The $3 \mathrm{~nm}$ spherical indenter velocity was $2.5 \mathrm{~m} / \mathrm{s}$ and the interatomic potential of Voter et al. ${ }^{28}$ was used, with the substrate represented by BC-1. Only atoms with centrosymmetry parameter greater than 3 are shown. 


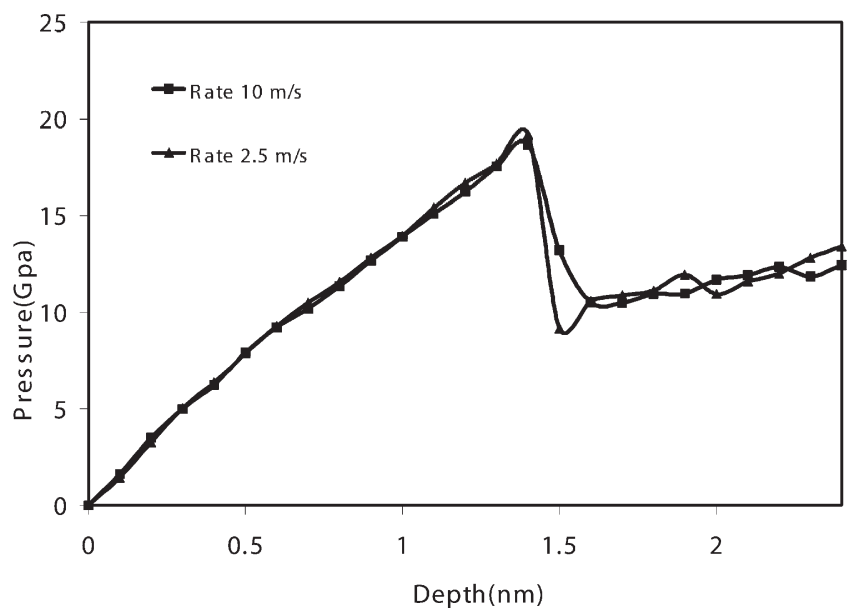

FIG. 3. Comparison of pressure curves for two different indentation velocities of a $30 \mathrm{~nm}$ spherical indenter in a $20-\mathrm{nm} \mathrm{Ni}$ thin film. The interatomic potential of Voter et al. $^{28}$ was used, with the substrate represented by $\mathrm{BC}-1$.

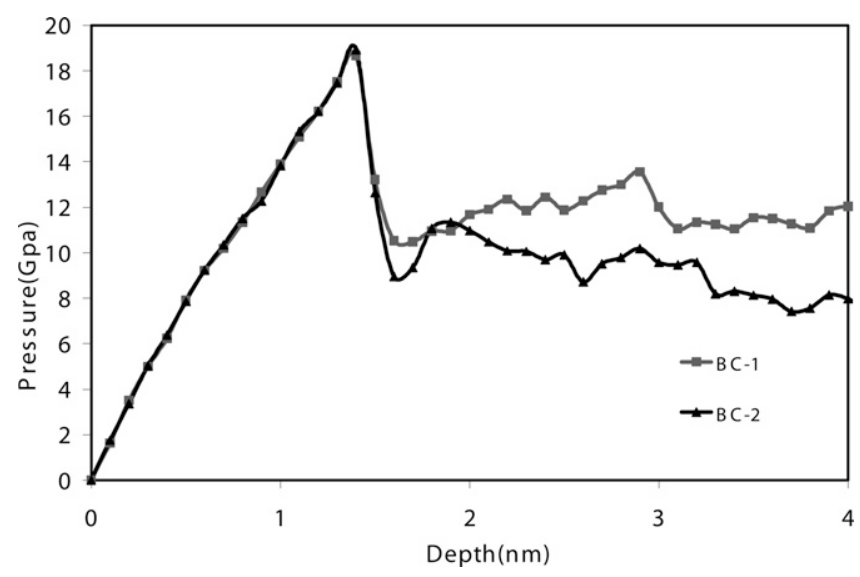

FIG. 4. Comparison of pressure versus depth curves for two different boundary conditions for a $20-\mathrm{nm} \mathrm{Ni}$ thin film. The interatomic potential of Voter et al. ${ }^{28}$ was used with a $30 \mathrm{~nm}$ spherical indenter at $10 \mathrm{~m} / \mathrm{s}$.

pressure curves start to deviate at about $15 \%$ of the film thickness. This implies that the substrate is having an effect on the indentation process at such depths. Similar substrate effects have been observed by Saha and Nix ${ }^{33}$ where the hardness has an effect on the substrate chosen for nanoindentation. This is also consistent with the experiments where the maximum depth of penetration is restricted to $10 \%$ of the film thickness, although research suggests that this rule has no physical basis. ${ }^{34}$

\section{Effects of the indenter radius}

To study the effect of different radii in nanoindentation on the obtained hardness values, the pressure versus displacement curves are plotted for three different radiuses of $7.5,15$, and $30 \mathrm{~nm}$ along with the experimental results. Figure 5 shows that the stress required to emit the first dislocation is independent of the radius of the

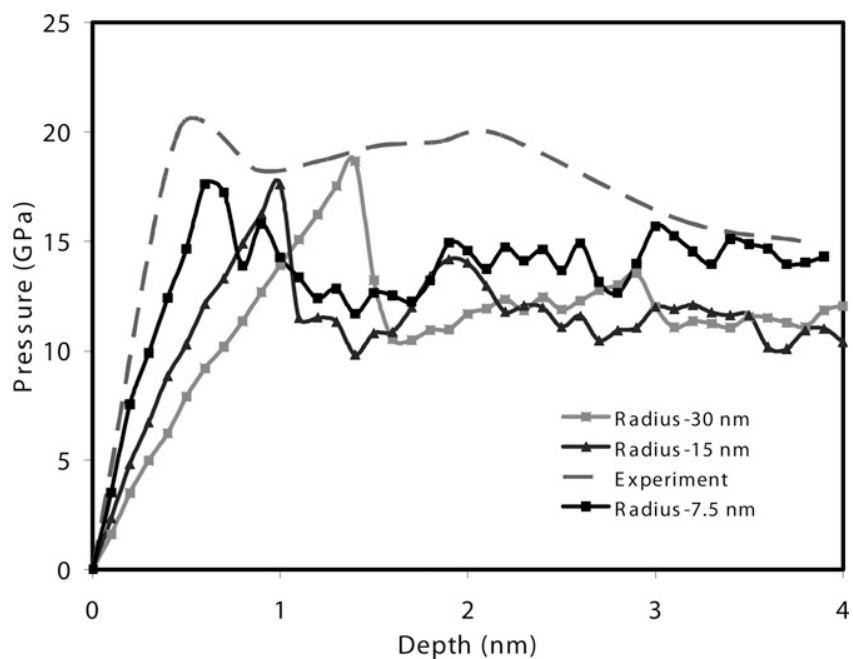

FIG. 5. Comparison of pressure versus depth for different radii for the $20-\mathrm{nm} \mathrm{Ni}$ film. The interatomic potential of Voter et al. ${ }^{28}$ was used with a $30 \mathrm{~nm}$ spherical indenter and $\mathrm{BC}-1$.

indenter. The elastic region shows a radius effect because the pressure has an inverse square root relation with the radius of the indenter, as defined by the Hertzian theory. ${ }^{35}$ As the indentation depth increases, the effect of different radii becomes more obvious, due to substrate effects. Indentation hardness tests done in submicron scale by $\mathrm{Lu}$ and $\mathrm{Bogy}^{36}$ using finite elements show that for an aluminum layer on a (harder) silicon substrate, the hardness is relatively insensitive to the tip radius. Figure 5 also shows the experimental results obtained for the same film orientation and thickness and a 50-nm Berkovich indenter. Note that despite the different shape of the indenter, the maximum stress values obtained from both simulations and experiments agree within about $15 \%$. The simulations show that as the indenter radius increases, the initial slope of the pressure curve is smaller and the depth at which maximum pressure is reached increases. Even if the experimental curve has the largest indenter radius, its slope is the highest and reaches a maximum pressure at the smallest indentation depth. This difference could be because the indenter tip is not perfectly spherical in the experiments. Indeed, the shape of the indenter can make a significant difference $^{34-36}$ because the contact area evolution, as the indentation proceeds, will depend on the indenter shape Another possible explanation for this discrepancy is the role played by pre-existing defects in the experimental film.

\section{Effects of different interatomic potential used}

The interatomic potentials used in these massively parallel large scale simulations are necessarily empirical in nature. The empirical potentials are developed based on known properties of the material, but questions remain related to the transferability of these potentials to 


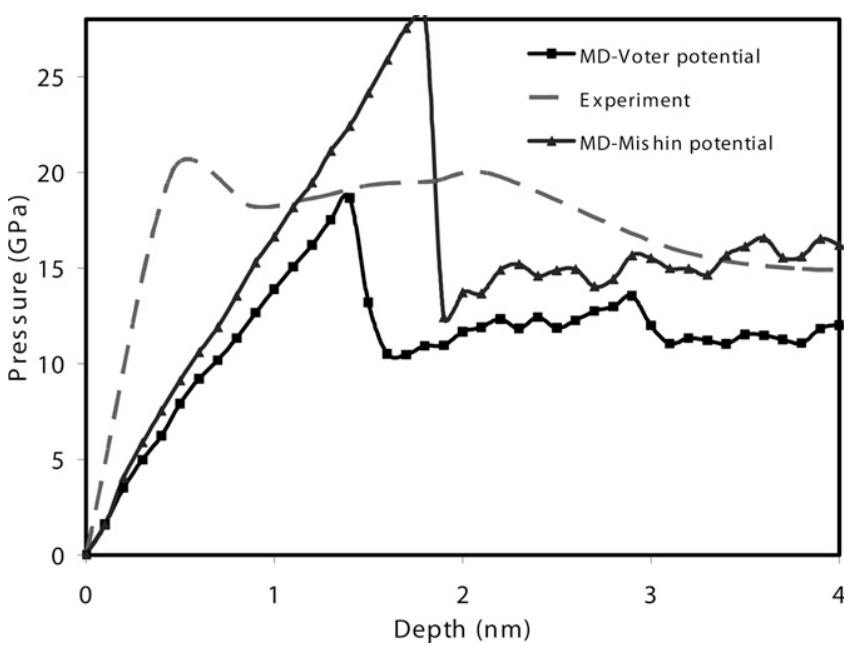

FIG. 6. Comparison of experimental results with Voter et al. ${ }^{28}$ and Mishin et al. ${ }^{27}$ potentials for $20-\mathrm{nm}$ Ni film. A $30 \mathrm{~nm}$ spherical indenter was used at $10 \mathrm{~m} / \mathrm{s}$ and the boundary conditions are BC-1.

situations that are far from equilibrium, such as those occurring during nanoindentation. In the present work we tested two different empirical interatomic potentials, both within the framework of the embedded atom method. The purpose of the comparison is to study the influence of the particular potential chosen and to see which interatomic potential mimics the experimental results best. Figure 6 shows the pressure versus displacement curves obtained with the two interatomic potentials for Ni. The areas used for the computation of pressure for both simulations and experiment are given by Eq. (3). Comparing the stress, at which the first dislocation is emitted, the Voter-Chen potential gives a value of approximately $19 \mathrm{GPa}$ and the experimental result is 20 GPa. The Mishin potential, however, gives a higher hardness of $27 \mathrm{GPa}$. This is consistent with Nair et al. ${ }^{37}$ where single crystal $\mathrm{Ni}$ thin films showed a hardness value of $28 \mathrm{GPa}$ using the Mishin potential. Comparing the pressure values at a larger indentation depth, for example at $4 \mathrm{~nm}$, the Voter-Chen potential gives a value of about $12 \mathrm{GPa}$ and the experimental result is $15 \mathrm{GPa}$. The Mishin potential gives a value of about $16 \mathrm{GPa}$. The results do point out differences of up to $30 \%$, depending on the particular interatomic potential that is used. Zimmerman et al. ${ }^{38}$ have shown that the Mishin potential has considerably higher values of both intrinsic and unstable stacking fault energies than the Voter potential does. The values for the intrinsic stacking fault are 129 $\mathrm{mJ} / \mathrm{m}^{2}$ for the Mishin potential and $62 \mathrm{~mJ} / \mathrm{m}^{2}$ for the Voter potential. For the unstable stacking fault the Mishin potential gives $405 \mathrm{~mJ} / \mathrm{m}^{2}$, whereas the Voter potential gives $273 \mathrm{~mJ} / \mathrm{m}^{2}$. The unstable stacking fault is known to be related to the barriers for dislocation emission, and therefore plasticity. The differences may help explain some of the indentation behavior observed in Fig. 6 for both potentials.
These results are inconclusive as far as which potential better reproduces the experiments, with one potential reproducing the peak value better, and the other giving better agreement at a larger indentation depth. This is despite the fact that the Mishin potential has been developed on the basis of first principle calculations for metastable phases and was fitted to many more parameters compared to Voter potential.

Despite these uncertainties the comparison of simulation and experimental results points out the fact that the basic mechanisms of deformation can be captured in the simulation and good agreement can be obtained, within the uncertainty of empirical interatomic potentials.

\section{SUMMARY AND CONCLUSIONS}

In this work, we simulated the indentation of $\mathrm{Ni}$ [111] thin films of $20 \mathrm{~nm}$ thicknesses using molecular dynamics. The simulations addressed a comparative analysis with experimental results, performed for the same film orientation and thickness, and a similar indenter radius. Several important conclusions can be drawn from our results.

(1) The high indenter velocity does not affect the hardness value in a significant manner.

(2) Different boundary conditions used to represent the substrate do not affect the simulation results for depths up to $15 \%$ of the film thickness.

(3) Different radii used for indentation has no effect on the hardness value obtained for radii between 7.5 to $30 \mathrm{~nm}$.

(4) The most important source of uncertainty found in the simulations is related to the particular empirical interatomic potential used. Differences of $\sim 20 \%$ can be attributed to the interatomic potential.

Overall, our results indicate that simulations can be performed for sizes that are similar to experiments and that the results compare favorably. The most important source of error is the reliability of the interatomic potential description of the material.

Our simulations also show the deformation mechanism during indentation as controlled by dislocation emission, interaction, and motion. The present simulations are performed in single crystals, whereas the experimental films are expected to have defects that can act as nucleation sites. In previous work ${ }^{37}$ we have studied the effects of pre-existing defects in the form of low angle boundaries. We have found that the basic deformation mechanism remains the same. The low angle grain boundaries can act as dislocation sources under indentation. For the films with pre-existing defects we observed hardness of around $15 \mathrm{GPa}$, which are slightly lower than those observed here for single crystals. These results can give an estimate of the influence of pre-existing defects on observed hardness values. 


\section{ACKNOWLEDGMENTS}

This work was supported by National Science Foundation (NSF), Civil and Mechanical Systems, and Materials Theory. We also acknowledge Dr. Ronald Kriz for very valuable discussions. The simulations were performed using Virginia Tech's supercomputing facility (System X).

\section{REFERENCES}

1. A.C. Fischer-Cripps: Nanoindentation (Springer, New York, 2002).

2. W.W. Gerberich, J.C. Nelson, E.T. Lilleodden, P. Anderson, and J.T. Wyrobek: Indentation induced dislocation nucleation: The initial yield point. Acta Mater. 44(9), 3585 (1996).

3. D. Christopher, R. Smith, and A. Richter: Atomistic modelling of nanoindentation in iron and silver. Nanotechnology 12(3), 372 (2001).

4. J. Li, K.J. Van Vliet, T. Zhu, S. Yip, and S. Suresh: Atomistic mechanisms governing elastic limit and incipient plasticity in crystals. Nature 418(6895), 307 (2002).

5. E.T. Lilleodden, J.A. Zimmerman, S.M. Foiles, and W.D. Nix: Atomistic simulations of elastic deformation and dislocation nucleation during nanoindentation. J. Mech. Phys. Solids 51(5), 901 (2003).

6. Y.M. Lee, J.Y. Park, S.Y. Kim, S. Jun, and S. Im: Atomistic simulations of incipient plasticity under A1(111) nanoindentation. Mech. Mater. 37(10), 1035 (2005).

7. X.L. Ma and W. Yang: Molecular dynamics simulation on burst and arrest of stacking faults in nanocrystalline $\mathrm{Cu}$ under nanoindentation. Nanotechnology 14(11), 1208 (2003).

8. B. Shiari, R.E. Miller, and W.A. Curtin: Coupled atomistic/discrete dislocation simulations of nanoindentation at finite temperature. J. Eng. Mater. Technol. 127(4), 358 (2005).

9. R.A. Iglesias and E.P.M. Leiva: Two-grain nanoindentation using the quasicontinuum method: Two-dimensional model approach. Acta Mater. 54(10), 2655 (2006).

10. K.J. Kim, J.H. Yoon, M.H. Cho, and H. Jang: Molecular dynamics simulation of dislocation behavior during nanoindentation on a bicrystal with a Sigma $=5$ (210) grain boundary. Mater. Lett. 60 (28), 3367 (2006).

11. I. Szlufarska: Atomistic simulations of nanoindentation. Mater. Today 9(5), 42 (2006).

12. C.A. Schuh: Nanoindentation studies of materials. Mater. Today 9(5), 32 (2006).

13. P. Parakala, R.A. Mirshams, S. Nasrazadani, and K. Lian: Effects of thickness and indenter geometry in nanoindentation of nickel thin films, in Thin Films-Stresses and Mechanical Properties X, edited by S.G. Corcoran, Y-C. Joo, N.R. Moody, and Z. Suo (Mater. Res. Soc. Symp. Proc. 795, Warrendale, PA, 2004), pp. 355-360.

14. W.D. Nix and H.J. Gao: Indentation size effects in crystalline materials: A law for strain gradient plasticity. J. Mech. Phys. Solids 46(3), 411 (1998).

15. Y. Huang, F. Zhang, K.C. Hwang, W.D. Nix, G.M. Pharr, and G. Feng: A model of size effects in nano-indentation. J. Mech. Phys. Solids 54(8), 1668 (2006).

16. F. Zhang, R. Saha, Y. Huang, W.D. Nix, K.C. Hwang, S. Qu, and M. Li: Indentation of a hard film on a soft substrate: Strain gradient hardening effects. Int. J. Plast. 23(1), 25 (2007).

17. M.J. Cordill, W.M. Mook, A.K. Nair, D. Farkas, and W.W. Gerberich: Novel routes to nanocrystalline mechanical characterization. JOM 59(9), 59 (2007).

18. S.H. Chen, L. Liu, and T.C. Wang: Size dependent nanoindentation of a soft film on a hard substrate. Acta Mater. 52(5), 1089 (2004).
19. L.H. Li, L. Yin, and P.K. Chu: Finite element analysis of residual stress and interlayer in hard coating/interlayer/soft substrate system during nanoindentation. J. Mater. Res. 23(5), 1358 (2008).

20. A.K. Nair, D. Farkas, and R.D. Kriz: Molecular dynamics study of size effects and deformation of thin films due to nanoindentation. Comput. Model. Eng. Sci. 24(2-3), 239 (2008).

21. A. Khatibi and B. Mortazavi: A study on the nanoindentation behaviour of single crystal silicon using hybrid MD-FE method. Frontiers Mater. Sci. Technol. 32, 259 (2008).

22. J.M. Meza, F. Abbes, and M. Troyon: Penetration depth and tip radius dependence on the correction factor in nanoindentation measurements. J. Mater. Res. 23(3), 725 (2008).

23. S. Basu, A. Moseson, and M.W. Barsoum: On the determination of spherical nanoindentation stress-strain curves. J. Mater. Res. 21, 2628 (2006)

24. A.J. Moseson, S. Basu, and M.W. Barsoum: Determination of the effective zero point of contact for spherical nanoindentation. J. Mater. Res. 23, 204 (2008).

25. S. Basu and M.W. Barsoum: Deformation micromechanisms of $\mathrm{ZnO}$ single crystals as determined from spherical nanoindentation stress-strain curves. J. Mater. Res. 22, 2470 (2007).

26. S. Plimpton: Fast parallel algorithms for short-range moleculardynamics. J. Comput. Phys. 117(1), 1 (1995). Available at: http:// lammps.sandia.gov.

27. Y. Mishin, D. Farkas, M.J. Mehl, and D.A. Papaconstantopoulos: Interatomic potentials for monoatomic metals from experimental data and ab initio calculations. Phys. Rev. B 59(5), 3393 (1999).

28. A.F. Voter and S.P. Chen: Accurate interatomic potentials for $\mathrm{Ni}$ and $\mathrm{Ni}_{3} \mathrm{Al}$, in Characterization of Defects in Materials, edited by R.W. Siegel, J.R. Weertman, and R. Sinclair (Mater. Res. Soc. Symp. Proc. 82, Pittsburgh, PA, 1987), p. 175.

29. M.S. Lund and C. Leighton: Design and performance of a molecular beam epitaxy system for metallic heterostructure deposition illustrated by a study of the controlled epitaxy of $\mathrm{Cu}(111) /$ $\mathrm{Al}_{2} \mathrm{O}_{3}(0001)$. J. Vac. Sci. Technol., A 22(5), 2027 (2004).

30. M.S. Daw and M.I. Baskes: Embedded-atom method-Derivation and application to impurities, surfaces, and other defects in metals. Phys. Rev. B 29(12), 6443 (1984).

31. M. Finnis: Interatomic Forces in Condensed Matter (Oxford University Press, Oxford, UK, 2003), pp. 129-186.

32. C.L. Kelchner, S.J. Plimpton, and J.C. Hamilton: Dislocation nucleation and defect structure during surface indentation. Phys. Rev. B 58(17), 11085 (1998).

33. R. Saha and W.D. Nix: Effects of the substrate on the determination of thin film mechanical properties by nanoindentation. Acta Mater. 50(1), 23 (2002).

34. J.L. Hay, M.E. O'Hern, and W.C. Oliver: The importance of contact radius for substrate-independent property measurement of thin films, in Fundamentals of Nanoindentation and Nanotribology, edited by N.R. Moody, W.W. Gerberich, N. Burnham, and S.P. Baker (Mater. Res. Soc. Symp. Proc. 522, Warrendale, PA, 1998), p. 27.

35. K.L. Johnson: Contact Mechanics (Cambridge University Press, Cambridge, UK, 1985).

36. C.J. Lu and D.B. Bogy: The effect of tip radius on nano-indentation hardness tests. Int. J. Solids Struct. 32(12), 1759 (1995).

37. A.K. Nair, E. Parker, P. Gaudreau, D. Farkas, and R.D. Kriz: Size effects in indentation response of thin films at the nanoscale: A molecular dynamics study. Int. J. Plast. 24(11), 2016 (2008).

38. J.A. Zimmerman, J.H. Gao, and F.F. Abraham: Generalized stacking fault energies for embedded atom FCC metals. Modell. Simul. Mater. Sci. Eng. 8(2), 103 (2000). 